\title{
Light pressure across all scales: editorial
}

\author{
Andrea Macchi ${ }^{1,2, \mathrm{a}}{ }_{\mathbb{D}}$, Onofrio M. Maragò $^{3, \mathrm{~b}}{ }_{\mathbb{D}}$ \\ ${ }^{1}$ CNR, National Institute of Optics (INO), Adriano Gozzini Research Unit, Via G. Moruzzi 1, Pisa, Italy \\ 2 Enrico Fermi Department of Physics, University of Pisa, Largo B. Pontecorvo 3, 56127 Pisa, Italy \\ ${ }^{3}$ CNR-IPCF, Istituto per i Processi Chimico-Fisici, v.le F. Stagno D’Alcontres 37, 98158 Messina, Italy
}

(C) The Author(s), under exclusive licence to Società Italiana di Fisica and Springer-Verlag GmbH Germany, part of Springer Nature 2021

\section{Introduction}

Light pressure was first recognized by Kepler [1] in its explanation of comets tail. Its understanding lies within the theory of electromagnetism by Maxwell [2] and the identification of light momentum associated to the Poynting vector [3]. The Italian physicist A. Bartoli independently predicted the pressure of light on the basis of thermodynamics $[4,5]$. Despite the experimental attempts at the beginning of the last century by Lebedev $[4,5]$ and Nichols and Hull [6], the advent of the laser [7] represented the revolutionary breakthrough that enabled the pioneering experiments by Ashkin and co-workers on radiation pressure [8, 9], optical trapping of atoms [10,11], particles [12], cells [13], viruses and bacteria [14]. The latter three experiments were carried out with the setup configuration commonly known as optical tweezers [15], a tightly focused beam of laser light capable of holding particles in three dimensions.

The Nobel Prize in Physics has recognized at least three times the pressure of laser light as an unique tool to control and manipulate matter: in 1997 S. Chu, C. Cohen-Tannoudji and W. D. Phillips were awarded for laser cooling which had an enormous impact in atomic physics [16], while in 2018 A. Ashkin was awarded for optical tweezers and D. Strickland with G. Mourou for chirped pulse amplification which allows to generate the most intense light pressure ever at a focus, yielding promise for compact particle accelerators [17, 18]. Looking at increasingly larger scales, light pressure has a key role in optomechanics [19] including the detection of supefluid light states [20], in gravitational interferometers [21, 22], and also in the recently rediscovered visionary concept of laser-boosted sail probes for interstellar travel [23, 24]. Indeed, the Universe offers several examples of cosmic phenomena with a dominant role of the pressure exerted from electromagnetic radiation.

This focus point issue aimed to collect new results, insights, and reviews on light pressure in different physical contexts. Because of the interdisciplinary character of the focus issue, each paper highlights general aspects more than technical details, in order to be accessible to a broad readership. Published manuscripts reflect the diversity of interest in the field. In our original plan we wished to cover even more physical contexts and applications where light pressure plays a pivotal role. Unfortunately, the issues and strain caused by the COVID-19

\footnotetext{
a e-mail: andrea.macchi@ino.cnr.it (corresponding author)

be-mail: onofrio.marago@cnr.it
} 
pandemic affected the number of contributions, as well as spread the submissions across 2020 and early 2021.

Srivastava and Swartzlander [25] investigate the optomechanics of a rigid non-spinning light sail that mitigates catastrophic sail walk-off and tumbling by use of a flat axicon diffraction grating. Light sail acceleration by ultrashort, superintense laser pulses is also investigated as an approach to compact accelerators of matter by Pegoraro et al. [26]. The theory and practice of optical tweezers and its applications to single-molecule experiments are reviewed by Pesce et al. [27] and by Zaltron et al. [28], respectively. Novel biophysical applications of optical tweezers are shown in the work by Sergides et al. [29], where they investigate the transduction of optical forces in living cells and FRET-based molecular force microscopy.

Theory and modeling are crucial for a better understanding of light forces. In particular, transverse spin-dependent optical forces are the subject of the paper by Marques et al. [30] and Polimeno et. al [31], related with the unconventional properties of the Belinfante's spin momentum. The integration of optical tweezers with Raman spectroscopy, Raman tweezers, has opened to important applications in the spectroscopic characterization of biomolecules and cells that are experimentally investigated by Foti et al. [32] and Bernatova et al. [33], respectively.

Callegari et al. [34] review how optical tweezers have been successfully used to quantitatively study critical Casimir forces acting on particles in suspensions. Finally, Polimeno et al. (space tweezers collaboration) [35] describe how optical tweezers could be used to trap and characterize extraterrestrial particulate matter opening perspectives for optical tweezers applications in space, on planetary bodies surface, or in curator facilities designed for the uncontaminated handling and preliminary characterization of extraterrestrial samples returned by space probes.

In conclusion, we hope that the present focus point issue will encourage further experimental and theoretical work in these exciting and diverse fields, and possibly transferring hints and ideas across different areas. We would like to thank all the authors who have contributed to this issue, as well as all the anonymous reviewers who dedicated their time and expertise in the assessment of all submitted manuscripts. We offer special thanks to the Editors in-Chief of EPJ plus and to the publishing staff who supported this issue.

We wish to dedicate this Focus Point Issue to the memory of Arthur Ashkin, David Neely, Michael I. Mishchenko, and Juan José Sáenz, pioneers and great scientists, whose visionary contributions have opened unexplored paths in so many research fields.

The work of O.M.M. is supported by the agreement ASI-INAF n.2018-16-HH.0, project "SPACE Tweezers" and the MSCA ITN (ETN) project "ActiveMatter".

\section{References}

1. J. Kepler, De Cometis libelli tres (Avgvstae Vindelicorvm, A. Apergeri, 1619)

2. J.C. Maxwell, A Treatise on Electricity and Magnetism (Clarendon Press, Oxford, 1873).

3. J.H. Poynting, On the transfer of energy in the electromagnetic field. Philos. Trans. R. Soc. Lond. 175, 343 (1884)

4. A. Bartoli, Il calorico raggiante e il secondo principio di termodinamica. Il Nuovo Cimento 15, 196 (1884)

5. P. Lebedev, Untersuchungen über die druckkräfte des lichtes. Ann. Phys. 311, 433 (1901)

6. E.F. Nichols, G.F. Hull, A preliminary communication on the pressure of heat and light radiation. Phys. Rev. 13, 307 (1901)

7. C.H. Townes, How the Laser Happened: Adventures of a Scientist (Oxford University Press, Oxford, 2002).

8. A. Ashkin, Acceleration and trapping of particles by radiation pressure. Phys. Rev. Lett. 24, 156 (1970)

9. A. Ashkin, Atomic-beam deflection by resonance-radiation pressure. Phys. Rev. Lett. 25, 1321 (1970) 
10. S. Chu, L. Hollberg, J.E. Bjorkholm, A. Cable, A. Ashkin, Three-dimensional viscous confinement and cooling of atoms by resonance radiation pressure. Phys. Rev. Lett. 55, 48 (1985)

11. S. Chu, J.E. Bjorkholm, A. Ashkin, A. Cable, Experimental observation of optically trapped atoms. Phys. Rev. Lett. 57, 314 (1986)

12. A. Ashkin, J.M. Dziedzic, J.E. Bjorkholm, S. Chu, Observation of a single-beam gradient optical trap for dielectric particles. Opt. Lett. 11, 288 (1986)

13. A. Ashkin, J.M. Dziedzic, T. Yamane, Optical trapping and manipulation of single cells using infrared laser beams. Nature 330, 769 (1987)

14. A. Ashkin, J.M. Dziedzic, Optical trapping and manipulation of viruses and bacteria. Science 235, 1517 (1987)

15. P.H. Jones, O.M. Maragò, G. Volpe, Optical Tweezers: Principles and Applications (Cambridge University Press, Cambridge, 2015).

16. C.J. Foot, Atomic Physics (Oxford University Press, Oxford, 2005).

17. E. Esarey, C.B. Schroeder, W.P. Leemans, Physics of laser-driven plasma-based electron accelerators. Rev. Mod. Phys. 81, 1229 (2009)

18. A. Macchi, M. Borghesi, M. Passoni, Ion acceleration by superintense laser-plasma interaction. Rev. Mod. Phys. 85, 751 (2013)

19. M. Aspelmeyer, T.J. Kippenberg, F. Marquardt, Cavity optomechanics. Rev. Mod. Phys. 86, 1391 (2014)

20. P.-É. Larré, I. Carusotto, Optomechanical signature of a frictionless flow of superfluid light. Phys. Rev. A 91, 053809 (2015)

21. P.R. Saulson, Fundamentals of Interferometric Gravitational Wave Detectors, 2nd edn. (World Scientific, Singapore, 2017).

22. L. Barsotti, J. Harms, R. Schanbel, Squeezed vacuum states of light for gravitational wave detectors. Rep. Prog. Phys. 82, 016905 (2018)

23. H.A. Atwater, A.R. Davoyan, O. Ilic, D. Jariwala, M.C. Sherrott, C.M. Went, W.S. Whitney, J. Wong, Materials challenges for the Starshot lightsail. Nat. Mater. 17, 861 (2018)

24. D. Kipping, Relativistic light sails. Astron. J. 153, 277 (2018)

25. P.R. Srivastava, G.A. Swartzlander, Optomechanics of a stable diffractive axicon light sail. EPJ Plus 135, $570(2020)$

26. F. Pegoraro, C. Livi, A. Macchi, Light sail boosted by instantaneous radiation pressure. EPJ Plus 136, 485 (2021)

27. G. Pesce, P.H. Jones, O.M. Maragò, G. Volpe, Optical tweezers: theory and practice. EPJ Plus 135, 949 (2020)

28. A. Zaltron, M. Merano, G. Mistura, C. Sada, F. Seno, Optical tweezers in single-molecule experiments. EPJ Plus 135, 896 (2020)

29. M. Sergides, L. Perego, T. Galgani, C. Arbore, F.S. Pavone, M. Capitanio, Probing mechanotransduction in living cells by optical tweezers and FRET-based molecular force microscopy. EPJ Plus 136, 316 (2021)

30. M.I. Marqués, S. Edelstein, P.A. Serena, A proposal to measure Belinfante's curl of the spin optical force based on the Kerker conditions. EPJ Plus 136, 185 (2021)

31. P. Polimeno, M.A. Iatì, C. DegliEspostiBoschi, S.H. Simpson, V. Svak, O. Brzobohatý, P. Zemánek, O.M. Maragò, R. Saija, T-matrix calculations of spin-dependent optical forces in optically trapped nanowires. EPJ Plus 136, 86 (2021)

32. A. Foti, M.G. Donato, O.M. Maragò, P.G. Gucciardi, Optically induced aggregation by radiation pressure of gold nanorods on graphene for SERS detection of biomolecules. EPJ Plus 136, 30 (2021)

33. S. Bernatová, K. Rebrošová, Z. Pilát, M. Šerý, A. Gjevik, O. Samek, J. Ježek, M. Šiler, M. Kizovský, T. Klementová, V. Holá, Rapid detection of antibiotic sensitivity of Staphylococcus aureus by Raman tweezers. EPJ Plus 136, 233 (2021)

34. A. Callegari, A. Magazzù, A. Gambassi, G. Volpe, Optical trapping and critical Casimir forces. EPJ Plus 136, 213 (2021)

35. P. Polimeno, A. Magazzù et al., (Space tweezers collaboration), optical tweezers in a dusty universe. EPJ Plus 136, 339 (2021) 